\title{
THERMAL CREEP STRESS AND STRAIN ANALYSIS IN A NON-HOMOGENEOUS SPHERICAL SHELL
}

\author{
PANKAJ THAKUR \\ Department of Mathematics, Faculty of Science and Technology, ICFAI University Baddi, Solan, India \\ e-mail: pankaj_thakur15@yahoo.co.in; dr_pankajthakur@yahoo.com \\ SATYA BIR Singh \\ Department of Mathematics, Punjabi University Patiala, Punjab, India; e-mail: sbsingh69@yahoo.com \\ D.S. PATHANia \\ Department of Mathematics, Guru Nanak Dev Engineering College, Ludhiana, Punjab, India \\ e-mail: despathania@yahoo.com \\ Gaurav Verma \\ Research Scholar, IKG Punjab Technical University Kapurthala, Punjab, India; e-amil: gkdon85@gmail.com
}

\begin{abstract}
The purpose of this paper is to present study of thermal creep stress and strain rates in a non-homogeneous spherical shell by using Seth's transition theory. Seth's transition theory is applied to the problem of creep stresses and strain rates in the non-homogeneous spherical shell under steady-state temperature. Neither the yield criterion nor the associated flow rule is assumed here. With the introduction of thermal effect, values of circumferential stress decrease at the external surface as well as internal surface of the spherical shell. It means that the temperature dependent materials minimize the possibility of fracture at the internal surface of the spherical shell. The model proposed in this paper is used commonly as a design of chemical and oil plants, industrial gases and stream turbines, high speed structures involving aerodynamic heating.
\end{abstract}

Keywords: stress, strain rates, thermal, spherical shell, non-homogeneous

\section{Introduction}

Spherical shell structures have found widespread use in modern technology such as design of chemical and oil plants, accumulator shells, pressure vessel for industrial gases or media transportation of high-pressurized fluids and piping of nuclear containment, high speed structures involving aerodynamic heating, submerged undersea structures, earth sheltered domes, and the like. These spherical systems are effective from the perspectives of both structural and architectural design. In many of these cases, the spherical shells have to operate under severe mechanical and thermal loads causing significant creep and thus reducing its service life. The collapse or damage is initiated by creep, shrinkage and thermal effects, or from their interaction with structures that both experience or do not experience environmental degradation. Consequently, demand for strengthening and upgrading the existing concrete structures, because of damage caused by long-term effects and excessive structural deformations, has been recognized. However, before the application of costly strengthening techniques, understanding of nonlinear long-term behaviour of the existing and new spherical shells is essential, and the development of suitable and reliable theoretical approaches for their analysis and safety assessment is required. Creep effects generally increase deformations of a shell structure even under room temperatures, and are usually only considered to affect behaviour at the service ability limit states. Therefore, the analysis of 
long term steady state creep deformations of shells is very important in these applications (Hamed et al., 2010; Kashkoli and Nejad, 2014). Due to the occurrence of these creep deformations, non-homogeneous materials are widely used in the engineering applications. Non-homogeneous materials are a specific class of composite materials known as functionally graded materials (FGM) in which constituents are graded in one or more direction with continuous variation to achieve desired properties. The smooth grading of the constituents result in better thermal properties, higher fracture toughness, improved residual stress distribution and reduced stress intensity factors. These properties allow non-homogeneous structures to withstand high pressure under elevated thermal environment. Therefore, the analysis of non-homogeneity in the spherical shell through a mathematical model by taking one and all the complexities into consideration is the major concern of researchers (Kar and Panda, 2016). Some degree of non-homogeneity is present in a wide class of materials such as hot rolled metals, magnesium and aluminum alloys. Non-homogenity can also be introduced by a certain external field which is a thermal gradient material as the elastic moduli of the materials vary with temperature (Olszak, 1960). Penny (1967) obtained the effects of creep in spherical shells by analysis similar to the corresponding elastic ones described here. Miller (1995) evaluated solutions for stresses and displacements in a thick spherical shell subjected to internal and external pressure loads. You et al. (2005) presented an accurate model to carry out elastic analysis of thick-walled spherical pressure vessels subjected to internal pressure. Kellogg and King (1997) developed a finite element model of convection in a spherical axisymmetric shell that we use to simulate upwelling thermal plumes in the mantle. Thakur (2011) analyzed creep transition stresses of a thick isotropic spherical shell by finitesimal deformation under steady state of temperature and internal pressure by using Seth's transition theory. Seth's transition theory does not acquire any assumptions like the yield condition, incompressibility condition, and thus poses and solves a more general problem from which cases pertaining to the above assumptions can be worked out. This theory utilizes the concept of a generalized strain measure and asymptotic solution at critical points or turning points of differential equations defining the deformed field and has been successfully applied to a large number of problems (Seth, 1962, 1966; Thakur, 2011, 2014; Thakur et al., 2016, 2017). Seth (1962) defined the concept of generalized strain measures as

$$
e_{i i}=\int_{0}^{e_{i i}^{A}}\left(1-2 \stackrel{e_{i i}^{A}}{e^{\frac{n}{2}-1}} d \stackrel{A}{e_{i i}=\frac{1}{n}}\left[1-\left(1-2 e_{i i}^{A}\right)^{\frac{n}{2}}\right] \quad i=1,2,3\right.
$$

where $n$ is the measure and ${ }_{i}^{A}$ are the Almansi finite strain components. For $n=-2,-1,0,1,2$ it gives the Cauchy, Green Hencky, Swainger and Almansi measures respectively. Non-homogeneity in a spherical shell has been taken as the compressibility of the material as

$$
C=C_{0} r^{-k}
$$

where $a \leqslant r \leqslant b, C_{0}$ and $k$ are real constants.

\section{Governing equations}

We consider a spherical shell whose internal and external radii are $a$ and $b$, respectively, and is subjected to uniform internal pressure $p_{i}$ of gradually increasing magnitude and temperature $\Theta_{0}$ applied to the internal surface $r=a$ as shown in Fig. 1. The components of displacement in spherical co-ordinates are given by (Seth, 1962, 1966)

$$
u=r(1-\beta) \quad v=0 \quad w=0
$$




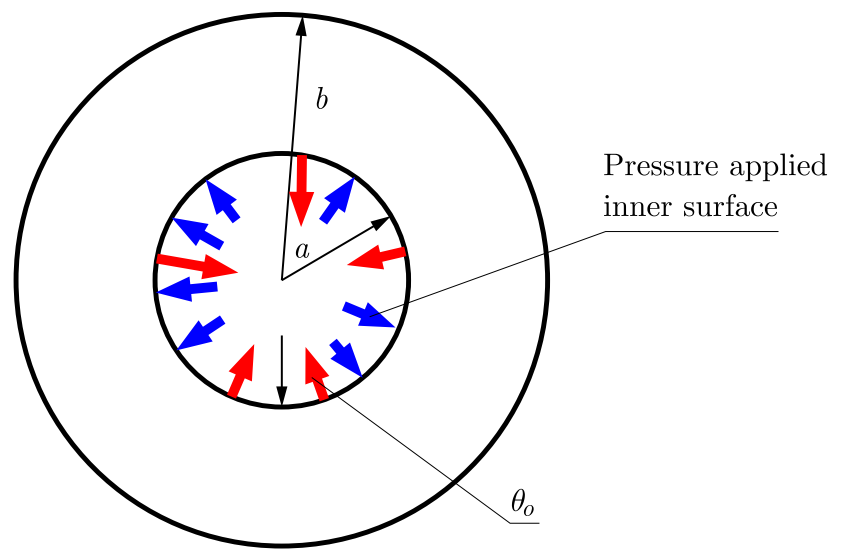

Fig. 1. Geometry of the spherical shell

where $u, v, w$ are displacement components, $\beta$ is a position function depending on $r$.

The generalized components of strain are given by Seth (1966)

$$
e_{r r}=\frac{1}{n}\left[1-\left(r \beta^{\prime}+\beta\right)^{n}\right] \quad e_{\theta \theta}=\frac{1}{n}\left(1-\beta^{n}\right)=e_{\varphi \varphi} \quad e_{r \theta}=e_{\theta \varphi}=e_{\varphi r}=0
$$

where $n$ is the measure and $\beta^{\prime}=d \beta / d r$.

Stress-strain relation. The stress-strain relations for a thermo-elastic isotropic material are given by (Parkus, 1976)

$$
T_{i j}=\lambda \delta_{i j} I_{1}+2 \mu e_{i j}-\xi \Theta \delta_{i j} T_{i j} \quad i, j=1,2,3
$$

where $T_{i j}$ are stress components and $e_{i j}$ is strain component, $\lambda$ and $\mu$ are Lame's constants, $I_{1}=e_{k k}$ is the first strain invariant, $\delta_{i j}$ is Kronecker's delta, $\xi=\alpha(3 \lambda+2 \mu)$, $\alpha$ being the coefficient of thermal expansion, and $\Theta$ is temperature. Further, $\Theta$ has to satisfy

$$
\nabla^{2} \Theta=0
$$

Substituting the strain components from Eq. (2.2) in Eq. (2.3), the stresses are obtained as

$$
\begin{aligned}
& T_{r r}=\frac{2 \mu}{n}\left[1-\left(r \beta^{\prime}+\beta\right)^{n}\right]+\frac{\lambda}{n}\left[3-\left(r \beta^{\prime}+\beta\right)^{n}-2 \beta^{n}\right]-\xi \Theta \\
& T_{\theta \theta}=T_{\varphi \varphi}=\frac{2 \mu}{n}\left(1-\beta^{n}\right)+\frac{\lambda}{n}\left[3-\left(r \beta^{\prime}+\beta\right)^{n}-2 \beta^{n}\right]-\xi \Theta \\
& T_{r \theta}=T_{\theta \varphi}=T_{\varphi r}=0
\end{aligned}
$$

Equation of equilibrium. The radial equilibrium of an element of the spherical shell requires

$$
\frac{d T_{r r}}{d r}+\frac{2}{r}\left(T_{r r}-T_{\theta \theta}\right)=0
$$

where $T_{r r}$ and $T_{\theta \theta}$ are the radial and hoop stresses, respectively.

Boundary conditions. The temperature satisfying Laplace equation (2.4) with boundary conditions

$$
\begin{aligned}
& \Theta=\Theta_{0} \wedge T_{r r}=-p_{i} \quad \text { at } \quad r=a \\
& \Theta=0 \wedge T_{r r}=0 \quad \text { at } \quad r=b
\end{aligned}
$$

where $\Theta_{0}$ is constant, is given by (Parkus, 1976)

$$
\Theta=\frac{\Theta_{0} \log (r / b)}{\log (a / b)}
$$


Critical points or turning points. Using Eq. (2.5) in Eq. (2.6), we get a non-linear differential equation in $\beta$ as

$$
\begin{gathered}
n P(P+1)^{n-1} \beta^{n+1} \frac{d P}{d \beta}=\left(\frac{\mu^{\prime}}{\mu}-\frac{C^{\prime}}{C}\right)\left\{3-2 C-\beta^{n}\left[2(1-C)+(1+P)^{n}\right]\right\}-2 C^{\prime} r\left(1-\beta^{n}\right) \\
-n \beta^{n} P\left[2(1-C)+(1+P)^{n}\right]+2 C \beta^{n}\left[1-(1+P)^{n}\right]-\frac{n C \bar{\Theta}_{0}}{2 \mu \beta^{n}}\left(\xi+r \xi^{\prime} \log \frac{r}{b}\right)
\end{gathered}
$$

where $\bar{\Theta}_{0}=\Theta_{0} / \log (a / b), C=2 \mu / \lambda+2 \mu$ and $r \beta^{\prime}=\beta P(P$ is a function of $\beta$ and $\beta$ is a function of $r$ only). The transition or turning points of $\beta$ in Eq. (2.9)) are $P \rightarrow-1$ and $P \rightarrow \pm \infty$.

\section{Analytical solution}

For finding thermal creep stresses and strain rates, the transition function is taken through the principal stress difference (see Seth, 1962, 1966; Thakur, 2011, 2014; Thakur et al., 2016, 2017) at the transition point $P \rightarrow-1$. We define the transition function $\psi$ as

$$
\psi=T_{r r}-T_{\theta \theta}=\frac{2 \mu \beta^{n}}{n}\left[1-(P+1)^{n}\right]
$$

where $\psi$ is a function of $r$ only, and $\psi$ is the dimension.

Taking logarithmic differentiation of Eq. (3.1) with respect to $r$ and substituting the value of $d P / d \beta$ from Eq. (2.9), we get

$$
\begin{aligned}
& \frac{d \log \psi}{d r}=\frac{n p}{r}+\frac{\mu^{\prime}}{\mu}-\frac{1}{r \beta^{n}\left[1-(1+P)^{n}\right]}\left[r\left(\frac{\mu^{\prime}}{\mu}-\frac{C^{\prime}}{C}\right)\left\{3-2 C-\beta^{n}\left[2(1-C)+(1+P)^{n}\right]\right\}\right. \\
& -2 r C^{\prime}\left(1-\beta^{n}\right)-n \beta^{n} P\left[2(1-C)+(1+P)^{n}\right] \\
& \left.\quad+2 C \beta^{n}\left[1-(1+P)^{n}\right]-\frac{n C \bar{\Theta}}{2 \mu}\left(\xi+r \xi^{\prime} \log \frac{r}{b}\right)\right]
\end{aligned}
$$

Taking asymptotic value of Eq. (3.2) at $P \rightarrow-1$, we get

$$
\frac{d}{d r}(\log \psi)=\frac{3 \mu^{\prime}}{\mu}-\frac{2 C^{\prime}}{C}-\frac{3 n}{r}+X
$$

where

$$
X=\frac{2(n-1) C}{r}-\frac{2 C \mu^{\prime}}{\mu}+\frac{2 C^{\prime}}{\beta^{n}}-\left(\frac{\mu^{\prime}}{\mu}-\frac{C^{\prime}}{C}\right) \frac{3-2 C}{\beta^{n}}+\frac{n C \bar{\Theta}_{0}}{2 \mu r \beta^{n}}\left(\xi+r \xi^{\prime} \log \frac{r}{b}\right)
$$

Integrating equation (3.3), we get

$$
\psi=A \frac{\mu^{3}}{C^{2} r^{3 n}} \exp (h)
$$

where $h=\int X d r$ and $A$ is a constant of integration, which can be determined from the boundary condition. From Eq. (3.1) and Eq. (3.4), we have

$$
T_{r r}-T_{\theta \theta}=A \frac{2 r \mu^{3}}{2 C^{2} r^{3 n+1}} \exp (h)=\frac{A r H}{2}
$$

where

$$
H=\frac{2 \mu^{3}}{C^{2} r^{3 n+1}} \exp (h)
$$


Substituting Eq. (3.5) in Eq. (2.6) and integrating, we get

$$
T_{r r}=B-A \int H d r
$$

where $B$ is a constant of integration, which can be determined from the boundary condition and asymptotic value of $\beta$ as $P \rightarrow-1$ is $D / r$, with $D$ being a constant.

Using boundary condition Eq. (2.7) in Eq. (3.6), we get

$$
A=-\frac{p_{i}}{\int_{a}^{b} H d r} \quad B=-\frac{p_{i}}{\int_{a}^{b} H d r} \int H d r
$$

where $p_{i}$ is pressure at the inner surface of the spherical shell. Using the integration constants $A$ and $B$ in Eq. (3.6), we get

$$
T_{r r}=\frac{p_{i}}{\int_{a}^{b} H d r} \int_{r}^{b} H d r
$$

Substituting Eq. (3.8) into Eq. (3.5), we get

$$
T_{\theta \theta}=T_{\varphi \varphi}=\frac{p_{i}}{\int_{a}^{b} H d r}\left(\int_{r}^{b} H d r+\frac{r H}{2}\right)
$$

We introduce non-homogeneity in the spherical shell due to variable compressibility as given in Eq. (1.2), then Eq. (3.8) and Eq. (3.9) become

$$
T_{r r}=\frac{p_{i}}{\int_{a}^{b} H d r} \int_{r}^{b} H_{1} d r \quad T_{\theta \theta}=T_{\varphi \varphi}=\frac{p_{i}}{\int_{a}^{b} H_{1} d r}\left(\int_{r}^{b} H_{1} d r+\frac{r H_{1}}{2}\right)
$$

where $T_{r r}, T_{\theta \theta}$ are radial and circumferential stresses, and

$$
\begin{aligned}
H_{1} & =\frac{2 \mu^{3}}{C^{2} r^{3 n+1}} \exp \left(h_{1}\right)=\frac{r^{-(3 n+k+1)}}{4\left(1-C_{0} r^{-k}\right)^{3}} \exp \left(h_{1}\right) \\
h_{1} & =-\frac{2(n-1)}{k} C_{0} r^{-k}-\frac{2 k C_{0} r^{n-k}}{D^{n}(n-k)}+\frac{k C_{0}}{D^{n}} \int \frac{r^{n-k-1}\left(3-2 C_{0}\right) r^{-k}}{1-C_{0}(b R)^{-k}} d r+2 \log \left(1-C_{0} r^{-k}\right) \\
& +\frac{\alpha n \bar{\Theta}_{0}}{D^{n}} \int\left(1-C_{0} r^{-k}\right)\left(3+\frac{C_{0} r^{-k}}{1-C_{0} r^{-k}}-\frac{k C_{0} r^{-k} \log (r / b)}{1-C_{0} r^{-k}}\right) r^{n-1} d r \\
C^{\prime} & =-k C_{0} r^{-k-1} \quad \frac{\mu^{\prime}}{\mu}=\frac{C^{\prime}}{C(1-C)}
\end{aligned}
$$

Equations (3.10) $)_{1,2}$ give thermal creep stresses for a spherical shell made of a non-homogeneous material under steady-state temperature. We introduce the following non-dimensional components as: $R=r / b, R_{0}=a / b, \sigma_{r r}=\tau_{r r} / p_{i}, \sigma_{\theta \theta}=\tau_{\theta \theta} / p_{i}$ and $\alpha \Theta_{0}=\Theta_{1}$. Equations $(3.10)_{1,2}$ in non-dimensional form become

$$
\sigma_{r r}=\frac{\int_{R}^{1} H_{2} d R}{\int_{R_{0}}^{1} H_{2} d r} \quad \sigma_{\theta \theta}=\sigma_{\varphi \varphi}=\frac{1}{\int_{R_{0}}^{1} H_{2} d R}\left(\int_{R}^{1} H_{2} d R+\frac{R H_{2}}{2}\right)
$$


where

$$
\begin{aligned}
H_{2} & =\frac{(b R)^{-(3 n+k+1)} C_{0} \lambda^{3}}{4\left(1-C_{0} b^{-k} R^{-k}\right)^{3}} \exp \left(h_{2}\right) \\
h_{2} & =-\frac{2(n-1)}{k} C_{0}(b R)^{-k}-\frac{2 k C_{0}(b R)^{n-k}}{D^{n}(n-k)} \\
& +\frac{k C_{0} b^{n-k}}{D^{n}} \int \frac{R^{n-k-1}\left(3-2 C_{0}\right) R^{-k}}{1-C_{0}(b R)^{-k}} d R+2 \log \left(1-C_{0} b^{-k} R^{-k}\right) \\
& +\frac{n \Theta_{1}}{D^{n} \ln R_{0}} \int\left(1-C_{0} b^{-k} R^{-k}\right)\left(3+\frac{C_{0}(b R)^{-k}}{1-C_{0} b^{-k} R^{-k}}-\frac{k C_{0}(b R)^{-k} \log R}{1-C_{0} b^{-k} R^{-k}}\right) R^{n-1} d R
\end{aligned}
$$

Particular case: In the absence of temperature gradient (i.e. $\left.\Theta_{1}=0\right)$, Eqs. (3.11), become

$$
\sigma_{r r}=\frac{\int_{R}^{1} H_{2}^{*} d R}{\int_{R_{0}}^{1} H_{2}^{*} d R} \quad \sigma_{\theta \theta}=\sigma_{\varphi \varphi}=\frac{1}{\int_{R_{0}}^{1} H_{2}^{*} d R}\left(\int_{R}^{1} H_{2}^{*} d R+\frac{R H_{2}^{*}}{2}\right)
$$

where

$$
\begin{aligned}
H_{2}^{*} & =\frac{(b R)^{-(3 n+k+1)} C_{0} \lambda^{3}}{4\left(1-C_{0} b^{-k} R^{-k}\right)^{3}} \exp \left(h_{2}^{*}\right) \\
h_{2}^{*} & =-\frac{2(n-1)}{k} C_{0}(b R)^{-k}-\frac{2 k C_{0}(b R)^{n-k}}{D^{n}(n-k)} \\
& +\frac{k C_{0} b^{n-k}}{D^{n}} \int \frac{R^{n-k-1}\left(3-2 C_{0}\right) R^{-k}}{1-C_{0}(b R)^{-k}} d R+2 \log \left(1-C_{0} b^{-k} R^{-k}\right)
\end{aligned}
$$

\section{Estimation of creep parameters}

When the creep sets in, the strains should be replaced by strain rates, then stress-strain relations are given (Sokolnikoff, 1946; Parkus, 1976)

$$
e_{i j}=\frac{1+\nu}{E} T_{i j}-\frac{\nu}{E} \delta_{i j} T+\alpha \Theta
$$

where $e_{i j}$ is the strain component and $T=T_{i i}$ is the first stress invariant and $\nu=(1-C) /(2-C)$ is Poisson's ratio. Differentiating Eq. (2.2) with respect to time $t$, we get

$$
\dot{e}_{\theta \theta}=-\beta^{n-1} \dot{\beta}
$$

For Swainger measure (i.e. $n=1$ ), Eq. (4.2) become

$$
\dot{\varepsilon}_{\theta \theta}=\dot{\beta}
$$

where $\dot{\varepsilon}_{\theta \theta}$ is the Swainger strain measure. From Eq. (3.1), the transition value $\beta$ is given at the transition point $P \rightarrow-1$ by

$$
\beta=\left(\frac{n}{2 \mu}\right)^{\frac{1}{n}}\left(T_{r r}-T_{\theta \theta}\right)^{\frac{1}{n}}
$$

Using Eqs. (4.2)-(4.4) in Eq. (4.1), we get

$$
\begin{array}{ll}
\dot{\varepsilon}_{r r}=m\left(\sigma_{r r}-\nu \sigma_{\theta \theta}+\alpha \Theta\right) & \dot{\varepsilon}_{\theta \theta}=m\left(\sigma_{\theta \theta}-\nu \sigma_{r r}+\alpha \Theta\right) \\
\dot{\varepsilon}_{\varphi \varphi}=-m\left[\nu\left(\sigma_{r r}+\sigma_{\theta \theta}\right)+\alpha \Theta\right] &
\end{array}
$$

where $\dot{\varepsilon}_{r r}, \dot{\varepsilon}_{\theta \theta}$ and $\dot{\varepsilon}_{\varphi \varphi}$ are strain rates, $\sigma_{r r}, \sigma_{\theta \theta}$ are stress components and $m=\left[n\left(\sigma_{r}-\sigma_{\theta}\right)(1+\nu)\right]^{\frac{1}{n}-1}$. These are the constitutive equations used by Odquist (1974) for finding the creep stresses, provided we put $n=1 / N$ and $N$ is the measure. 


\section{Numerical results and discussion}

For calculating creep stresses and strain rates on the basis of the above analysis, the following values have been taken, $\nu=0.5$ (incompressible material $C_{0}=0$ ), $\nu=0.42857$ (compressible material $C_{0}=0.25$ ) and 0.333 (compressible material $C_{0}=0.50$ ), $n=1 / 3,1 / 5,1 / 7$ (i.e. $N=3$, 5,7 ), thermal expansion coefficient $\alpha=5.0 \cdot 10^{-5} \mathrm{deg} \mathrm{F}^{-1}$ for Methyl Methacrylate (Levitsky and Shaffer, 1975) and $\Theta_{1}=\alpha \Theta_{0}=0$ and 0.5, $D=1$. In classical theory, the measure $N$ is equal to $1 / n$. The definite integrals in Eqs. (3.11) have been evaluated by using Simpson's rule. From Figs. 2-4, curves are presented between stresses along the radii ratio $R=r / b$ in the spherical shell made of compressible as well as incompressible materials for $k=-1,0,1$. It can be seen form Figs. 2 and 3 that the circumferential stresses are maximum at the external surface for $n=1 / 7$ and $k=-1,0$ for a compressible material as compared to the incompressible material. From Fig. 4, the circumferential stress is maximum at the internal surface for non-homogeneity $k=1$. The non-homogeneity increases the values of circumferential stress (i.e. $k=-1,0$ ), but reverse the result for $k=1$.

(a)

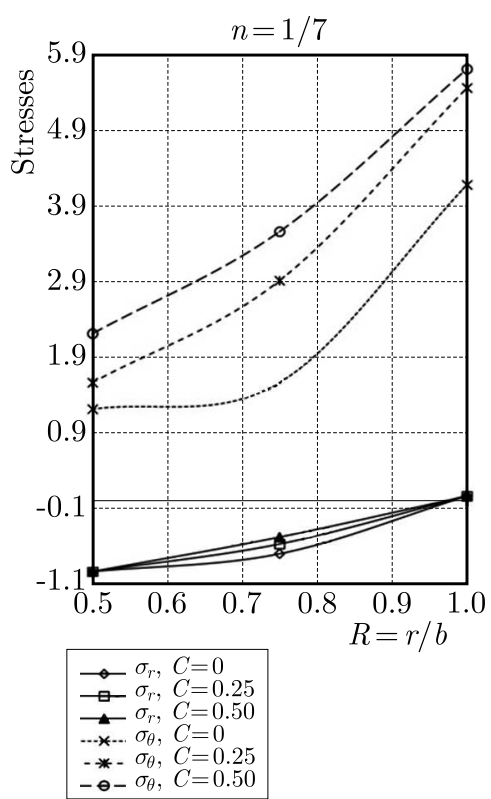

(b)

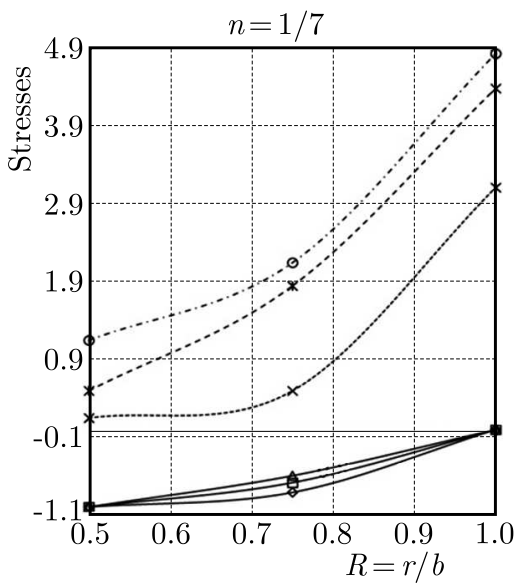

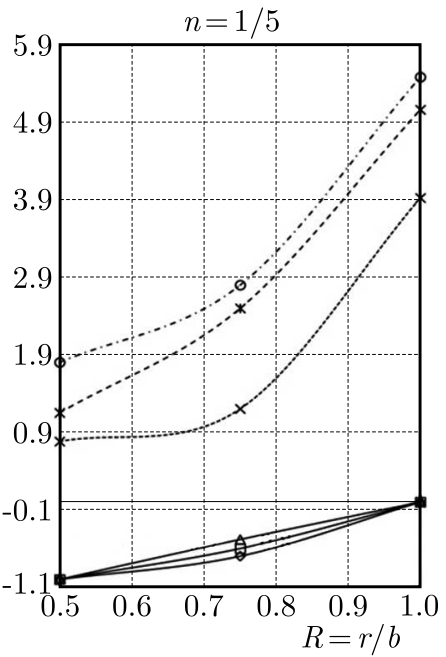
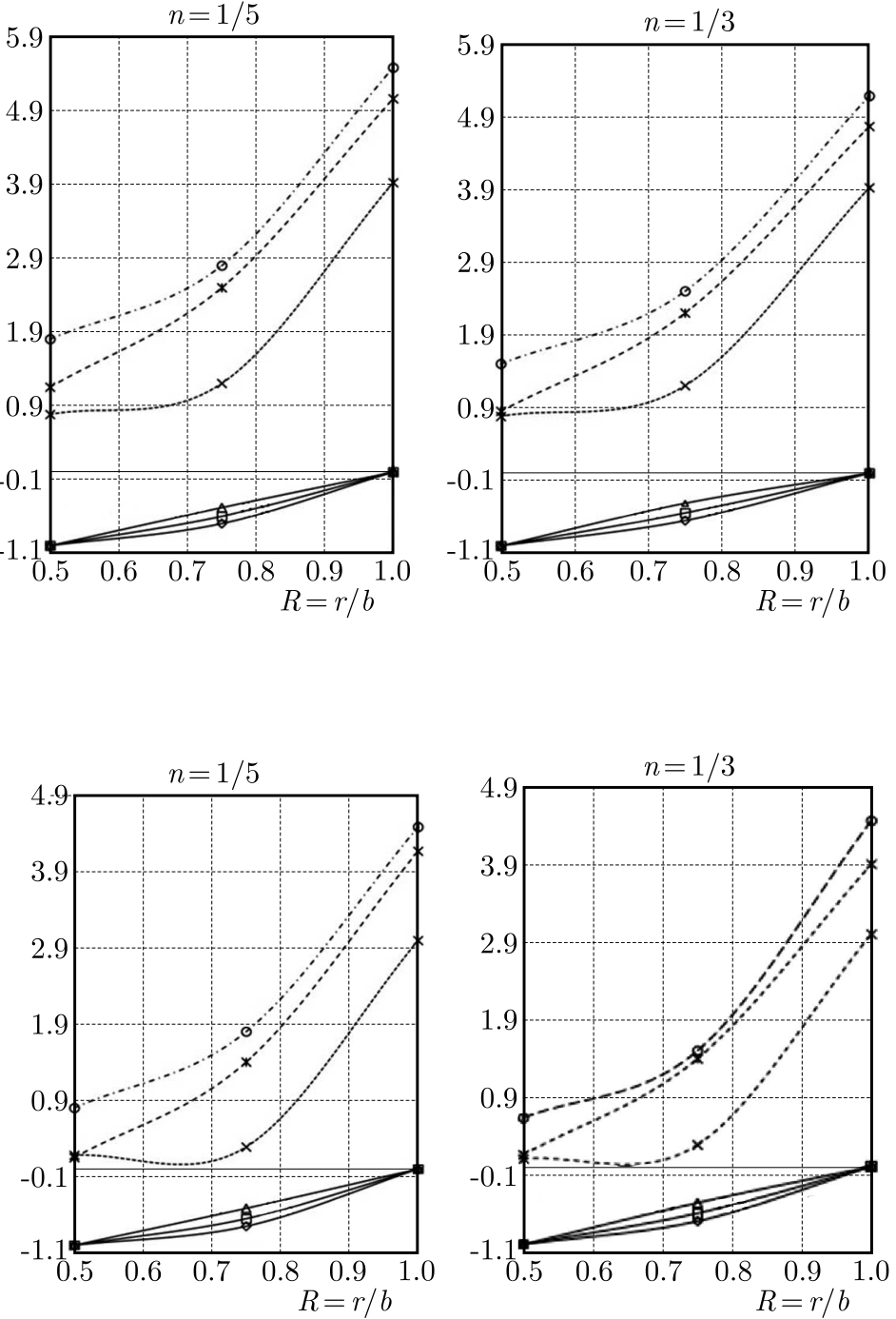

Fig. 2. Creep stresses in a non-homogeneous $(k=-1)$ spherical shell along the radii ratio $R=r / b$; (a) without temperature, (b) for temperature $\Theta_{1}=0.5$ 

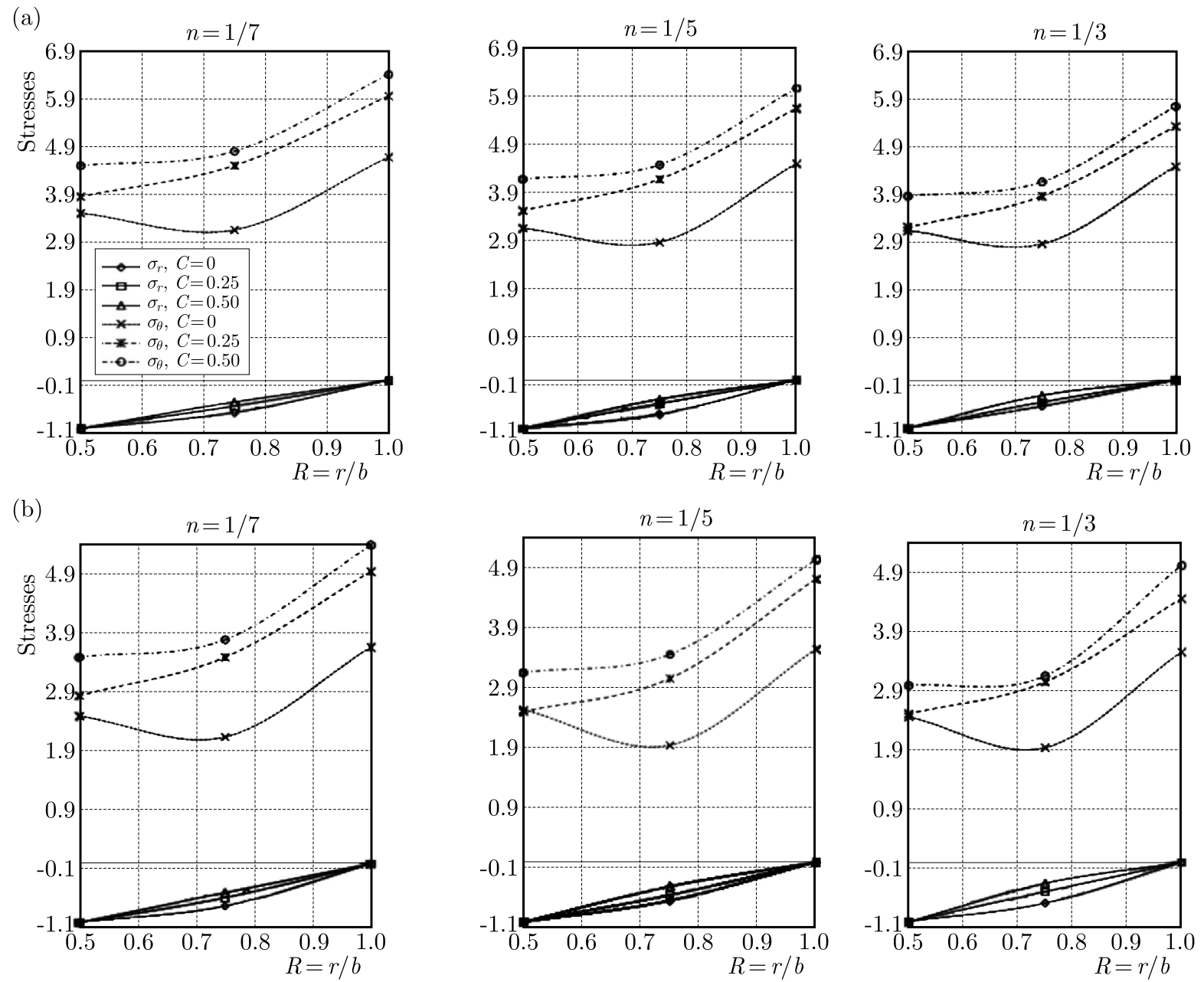

Fig. 3. Creep stresses in a non-homogeneous $(k=0)$ spherical shell along the radii ratio $R=r / b$; (a) without temperature, (b) for temperature $\Theta_{1}=0.5$

With the introduction of a temperature gradient, the values of circumferential stress are decreased at the external surface as well as internal surface of the spherical shell for different values of non-homogeneity. It means that temperature dependent materials minimize the possibility of fracture at the internal surface of the spherical shell. From (Fig. 5), curves are produced between strain rates along the radii ratio $R=r / b$ for the spherical shell made of compressible material $C=0.25$, i.e. saturated clay for $k=-1,0,1$. It can be seen that the strain rates are maximum at the external surface for $k=-1,0$ and reverse in the case $k=1$. With the introduction of a temperature gradient, the strain rates decrease at the internal surface as well as the external surface. It means that the temperature dependent materials minimize the possibility of fracture at the internal surface of the spherical shell.

\section{Acknowledgment}

The authors are grateful to the referee for his critical comments which led to a significant improvement of the paper.

\section{References}

1. Hamed E., Bradford M.A., Gilbert R.I., 2010, Nonlinear long-term behaviour of spherical shallow thin-walled concrete shells of revolution, International Journal of Solids and Structures, 47, 2, 204-215 
(a)

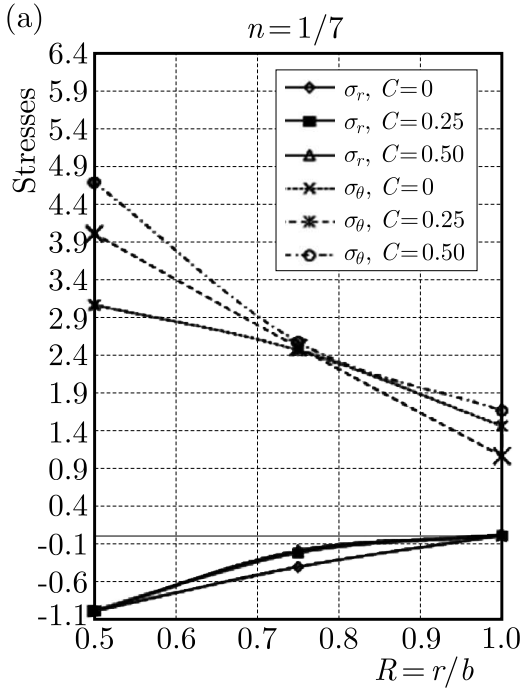

(b)

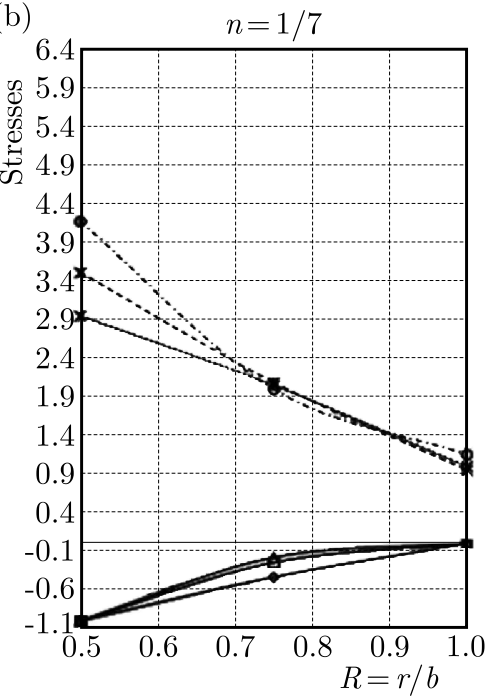

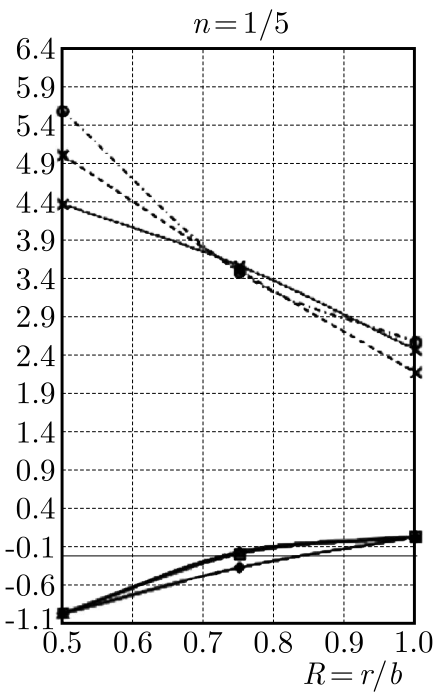
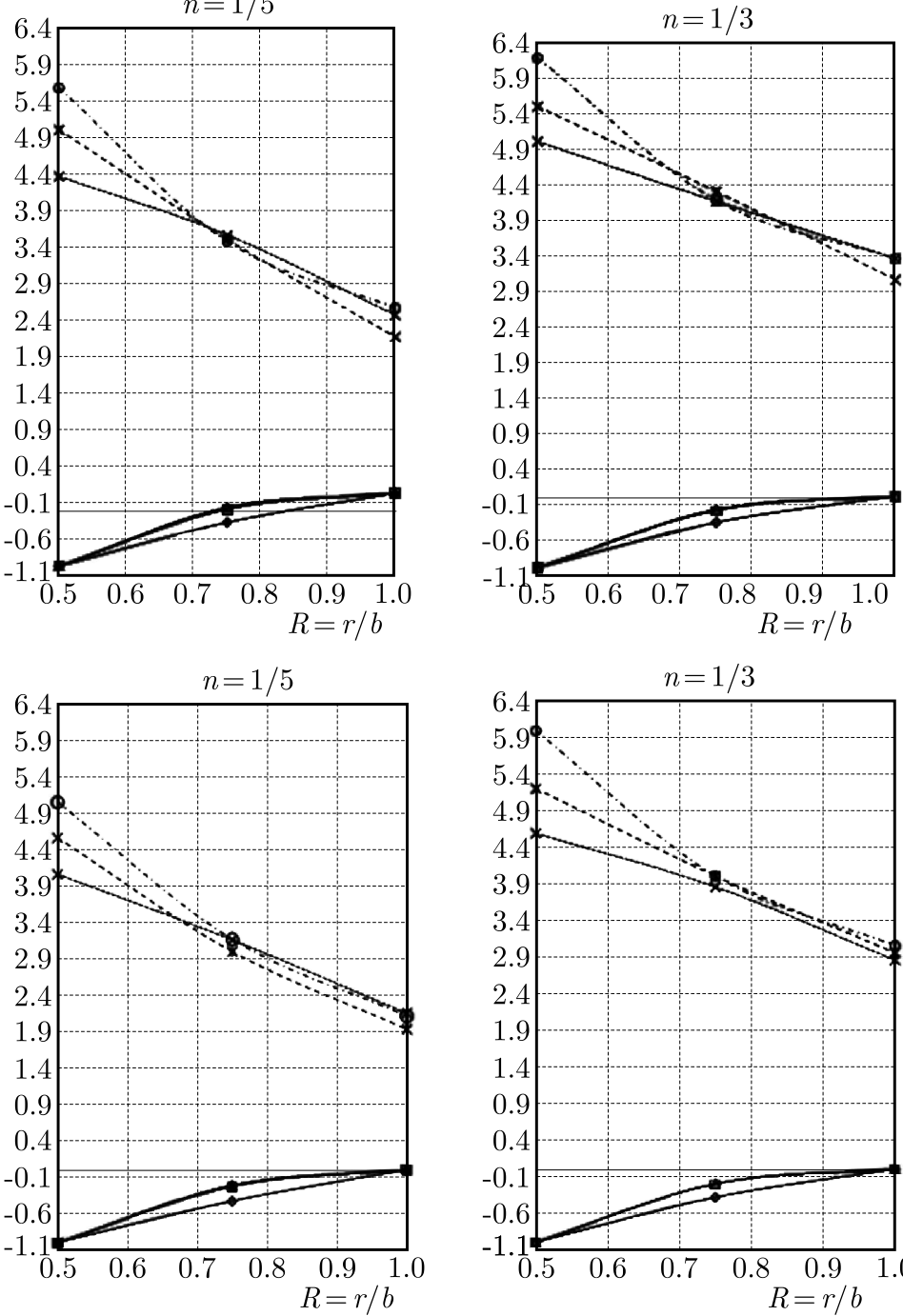

Fig. 4. Creep stresses in a non-homogeneous $(k=1)$ spherical shell along the radii ratio $R=r / b$; (a) without temperature, (b) for temperature $\Theta_{1}=0.5$

2. KAR V.R., PANDA S.K., 2016, Nonlinear thermo-mechanical deformation behaviour of P-FGM shallow spherical shell panel, Chinese Journal of Aeronautics, 29, 1, 173-183

3. Kashkoli M.D., Nejad M.Z., 2014, Effect of heat flux on creep stresses of thick walled cylindrical pressure vessels, Journal of Applied Research and Technology, 12, 3, 585-597

4. Kellogg L.H., King S.D., 1997, The effect of temperature dependent viscosity on the structure of new plumes in the mantle: Results of a finite element model in a spherical, axisymmetric shell, Earth and Planetary Science Letters, 148, 1/2, 13-26

5. Levitsky M., Shaffer, B.W., 1975, Residual thermal stresses in a solid sphere from a thermosetting material, Journal of Applied Mechanics, Transactions of ASME, 42, 3, 651-655

6. Miller G.K., 1995, Stresses in a spherical pressure vessel undergoing creep and dimensional changes, International Journal of Solids and Structures, 32, 14, 2077-2093

7. Odquist F.K.G., 1974, Mathematical Theory of Creep and Creep Rupture, Clarendon Press, Oxford

8. OlszaK W., 1960, Non-homogeneity in elasticity and plasticity, ZAMM, 40, 10/11, 522-523

9. PARkus H., 1976, Thermo-Elasticity, Springer-Verlag Wien, New York, USA 

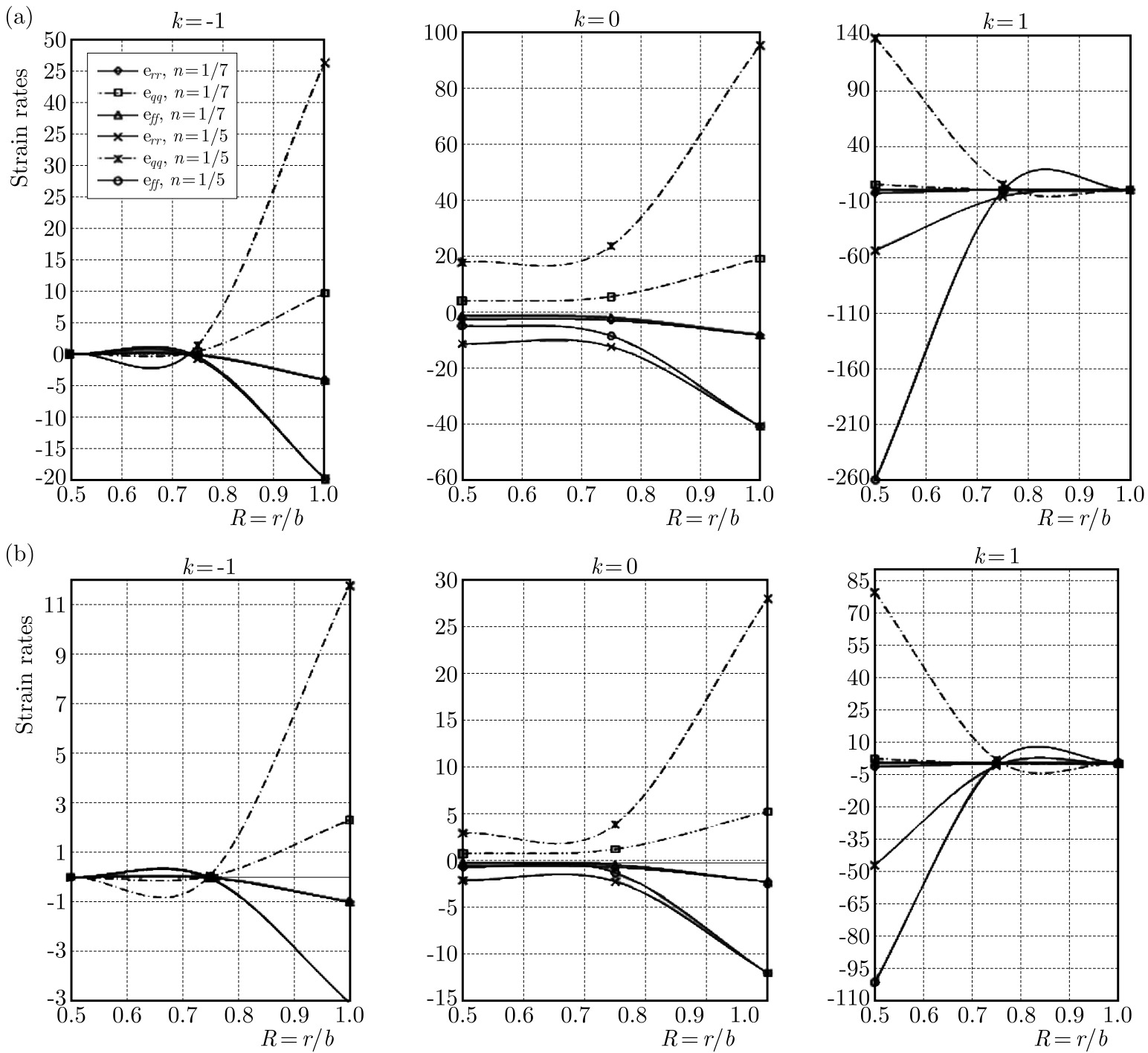

Fig. 5. Strain rates in a non-homogeneous spherical shell along the radii ratio $R=r / b$ for $C=0.25$; (a) without temperature $\Theta_{1}=0$, (b) for temperature $\Theta_{1}=0.5$

10. Penny R.K., 1967, The creep of spherical shells containing discontinuities, International Journal of Mechanical Sciences, 9, 6, 373-388

11. Seтн B.R., 1962, Transition theory of elastic-plastic deformation, creep and relaxation, Nature, 195, 896-897, DOI: 10.1038/195896a0

12. Sетн B. R., 1966, Measure concept in mechanics, International Journal of Non-Linear Mechanics, $\mathbf{1}, 1,35-40$

13. Sokolnikoff I.S., 1946, Mathematical Theory of Elasticity, 1st edition, Mc-Graw Hill Book Company, Inc., New York, 60-76

14. Thakur P., 2011, Creep transition stresses of a thick isotropic spherical shell by finitesimal deformation under steady state of temperature and internal pressure, Thermal Science, 15, 2, S157-S165

15. Thakur P., 2014, Steady thermal stress and strain rates in a circular cylinder with nonhomogeneous compressibility subjected to thermal load, Thermal Science, 18, 1, S81-S92

16. Thakur P., Singh S.B., Kaur J., 2016, Thermal creep stresses and strain rates in a circular disc with shaft having variable density, Engineering Computation, 33, 3, 698-712 
17. Thakur P., Gupta N., Singh S.B., 2017, Creep strain rates analysis in cylinder under temperature gradient for different material, Engineering Computations, 34, 3, DOI: 10.1108/EC-05-2016$-0159$

18. You L.H., Zhang J.J., You X.Y., 2005, Elastic analysis of internally pressurized thick-walled spherical pressure vessels of functionally graded materials, International Journal of Pressure Vessels and Piping, 82, 5, 347-354

Manuscript received December 2, 2016; accepted for print April 15, 2017 